\title{
NIL $K$-THEORY MAPS TO CYCLIC HOMOLOGY
}

\author{
CHARLES A. WEIBEL
}

\begin{abstract}
Algebraic $K$-theory breaks into two pieces: nil $K$-theory and Karoubi-Villamayor $K$-theory. Karoubi has constructed Chern classes from the latter groups into cyclic homology. We construct maps from nil $K$-theory to cyclic homology which are compatible with Karoubi's maps, but with a degree shift. Several recent results show that in characteristic zero our map is often an isomorphism.
\end{abstract}

Ever since A. Connes discovered cyclic homology in 1982, it has been widely held that cyclic homology is closely related to algebraic $K$-theory. Supporting this belief, Karoubi defined Chern classes $\lim c_{p}: K_{n}(A) \rightarrow \lim H C_{n+2 p}(A)$ in [K1], and Goodwillie defined a related map $\gamma: K_{n}(A) \rightarrow H C_{n}^{-}(A)$ in [G]. Other maps between $K$-theory and variants of cyclic homology have been constructed by Loday (unpublished), Burghelea [Bur 1], Ogle [Ogle 1], and others. Of course, all of these maps are related to the Dennis trace map to Hochschild homology, $D: K_{n}(A) \rightarrow H H_{n}(A)$, which R. K. Dennis discovered in 1975 [D].

In this paper, we prove three results. The first is that Karoubi's map factors through Goodwillie's.

THEOREM A. For every associative algebra, there is a commutative diagram:

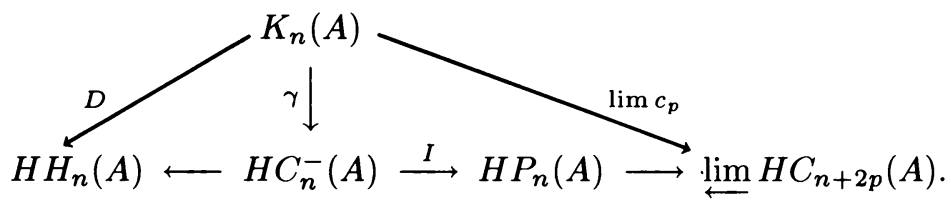

We will recall the definitions of $H C^{-}, H P$ and $H C$ in $\S 1$, and prove Theorem $\mathrm{A}$ in $\S 2$.

In order to explain the rest of our results, we need to decompose algebraic $K$ groups into two pieces: the Karoubi-Villamayor groups $K V_{*}$ and the nil $K_{*}$ groups, which may be thought of as the third terms in a long exact sequence

$$
\cdots K V_{n+1}(A) \rightarrow \operatorname{nil} K_{n}(A) \rightarrow K_{n}(A) \rightarrow K V_{n}(A) \cdots .
$$

When $A$ is regular, $K_{*}(A)=K V_{*}(A)$ and nil $K_{*}(A)=0$, so intuitively nil $K_{*}(A)$ measures the contribution to $K_{*}(A)$ coming from the singularities of $A$. The example $A=\mathbb{Z}$ shows that the abelian group structure of $K V_{*}(A)$ can be quite complicated. On the other hand, the nil $K$-groups are more predictable.

Received by the editors November $3,1986$.

1980 Mathematics Subject Classification (1985 Revision). Primary 18F25; Secondary 18G99, $19 \mathrm{D} 35$.

Key words and phrases. Cyclic homology, algebraic $K$-theory.

Partially supported by NSF grant. 
THEOREM B. If $\mathbb{Q} \subset A$ then nil $K_{n}(A)$ is a $\mathbb{Q}$-module for all $n$. On the other hand, if $p^{r}=0$ in $A$ for some $r$ then nil $K_{n}(A)$ is a $p$-group for all $n$.

For reasons of exposition, we prove this result in $\S 6$, because it requires tools unrelated to the main body of this paper.

Our third result is that, if we restrict to $\mathbb{Q}$-algebras, we can decompose Goodwillie's map into two pieces, introducing maps

$$
\operatorname{ch}: K V_{n}(A) \rightarrow H P_{n}(A), \quad \nu: \operatorname{nil} K_{n}(A) \rightarrow H C_{n-1}(A) .
$$

Before stating this result formally as Theorem $\mathrm{C}$, let us interpret the decomposition informally. We mean that Karoubi's map factors through ch in the sense that

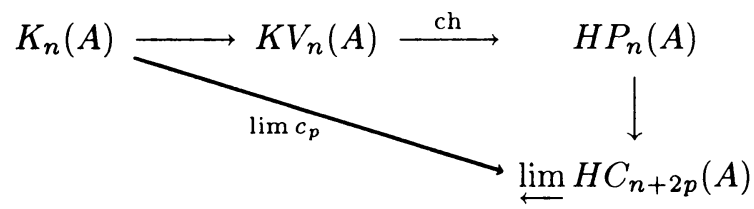

commutes, and $\nu$ lifts the Dennis trace map in the sense that

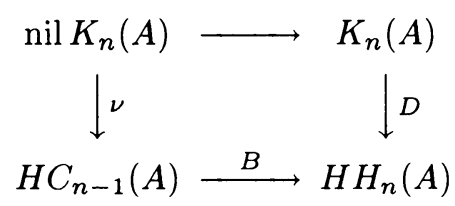

commutes. When $n=1$, the diagram $(0.2)$ is easy to understand.

EXAMPLE 0.3. The image of nil $K_{1}(A)$ in $K_{1}(A)=\mathrm{GL}(A) / E(A)$ is

$$
\operatorname{Unip}(A) / E(A)
$$

where $\operatorname{Unip}(A)$ is the subgroup of $\operatorname{GL}(A)$ generated be all unipotent matrices. The map

$$
\nu: \operatorname{nil} K_{1}(A) \rightarrow H C_{0}(A)=A /[A, A]
$$

factors through the trace of the logarithm map

$$
\ln (1+x)=x-\frac{1}{2} x^{2}+\frac{1}{3} x^{3} \cdots,
$$

in the sense that $\nu$ is the bottom row of the diagram

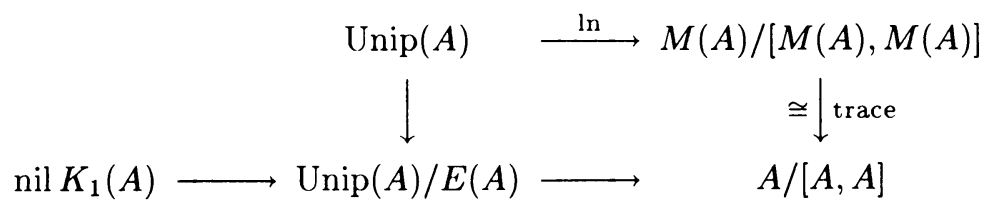

For example, let $A=\mathbb{Q}[t] /\left(t^{m}=0\right)$. Then $K_{1}(A)=\mathbb{Q}^{*} \oplus \operatorname{nil} K_{1}(A), \operatorname{nil} K_{1}(A)=$ $(1+t A)^{\times}$, and the map $B$ from $H C_{0}(A)=A$ to $H C_{1}(A)=\Omega_{A}$ is the usual derivative map $B(a)=d a$. If $x \in t A$ then $D(1+x)=(1+x)^{-1} d x=d(\ln (1+x))$, so by $(0.2)$ we see that $\nu(1+x)=\ln (1+x)$. In this case $\nu$ is the familiar isomorphism $\ln :(1+t A)^{\times} \cong t A$. In fact, by (6.3) this is the universal example of $\nu$ when $n=1$. 
The assertions (0.1) and (0.2) follow in general from

THEOREM C. For every $\mathbb{Q}$-algebra A, there is a commutative diagram with exact rows

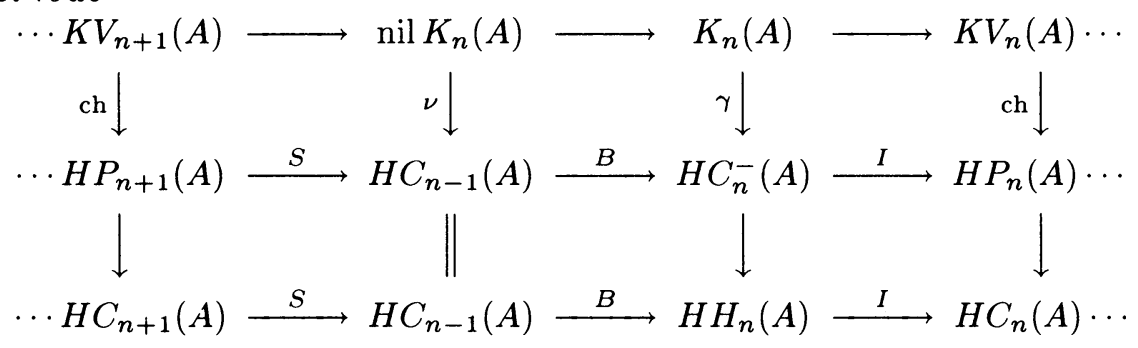

We will prove Theorem $\mathrm{C}$ in $\S 5$, after showing via examples in $\S 3$ that Theorem $\mathrm{C}$ cannot hold integrally. ( $\S 4$ is devoted to a translation of Theorem A into a topological setting that can be used to prove Theorem C.)

In the remainder of the introduction, I would like to explain why I think the map $\nu$ is fundamentally interesting. The first concrete hint that algebraic $K$-theory and cyclic homology were linked was the Loday-Quillen Theorem [LQ, 1.6]. If $A$ is an associative $\mathbb{Q}$-algebra and $\mathfrak{g l}(A)$ denotes the Lie algebra associated to GL $(A)$, this theorem states that

$$
H C_{n-1}^{\mathbb{Q}}(A) \cong \operatorname{Prim} H_{n}^{\mathrm{Lie}}(\mathfrak{g l}(A) ; \mathbb{Q})
$$

in analogy to the Milnor-Moore theorem, which asserts that

$$
K_{n}(A) \otimes \mathbb{Q} \cong \operatorname{Prim} H_{n}(\mathrm{GL}(A) ; \mathbb{Q}) .
$$

This hint already indicates the shift in indexing between $K$-theory and cyclic homology that we see in $\nu$. Based on this, R. Staffeldt was able in $[\mathbf{S}]$ to establish that if $A=R \oplus I, R$ the ring of integers in a number field and $I$ nilpotent, then

$$
K_{n}(A, I) \otimes \mathbb{Q} \cong H C_{n-1}^{\mathbb{Q}}(A \otimes \mathbb{Q}, I \otimes \mathbb{Q}) .
$$

Goodwillie spectacularly generalized this in $[\mathbf{G}]$, proving that if $A$ is a simplicial ring and $I$ a simplicial ideal with $\pi_{0}(I)$ nilpotent, then again there is an isomorphism (0.4). The key to this result was the construction of the map $\gamma$.

When $A$ is a $\mathbb{Q}$-algebra and $I$ a nilpotent ideal, Goodwillie's theorem tells us more. By [W2, p. 301] the groups $K V_{n}(A, I)$ vanish, so by Theorem B (or [W3, 1.4]) the groups $K_{n}(A, I)$ are $\mathbb{Q}$-vector spaces. In $[\mathbf{G} \mathbf{1}]$, Goodwillie proved that the $H P_{n}(A, I)$ vanish as well. Thus Goodwillie's result becomes

THEOREM (GOODWILLIE $[\mathbf{G}]$ ). When $I$ is a nilpotent ideal in a ring $A$, then $K_{n}(A, I) \otimes \mathbb{Q} \cong K_{n}(A \otimes \mathbb{Q} ; I \otimes \mathbb{Q})$. When $A$ is a $\mathbb{Q}$-algebra there are isomorphisms

$$
\begin{gathered}
\text { nil } K_{n}(A, I) \cong K_{n}(A, I) \\
\nu \downarrow \cong \quad \cong \downarrow \gamma \\
H C_{n-1}(A, I) \cong H C_{n}^{-}(A, I)
\end{gathered}
$$

Another result, joint work with C. Ogle [OW], concerns the so-called "excision situation": is an ideal of $A$, and $A \rightarrow B$ maps $I$ isomorphically onto an ideal of $B$. By [W2], the double relative groups $K V_{n}(A, B, I)$ are zero; when $A$ is a $\mathbb{Q}$-algebra, the groups $K_{n}(A, B, I)$ are $\mathbb{Q}$-vector spaces by Theorem B (or $[\mathbf{W} 3,1.5]$ ). Thus there is a map of $\mathbb{Q}$-vector spaces

$$
\nu: K_{n}(A, B, I) \cong \operatorname{nil} K_{n}(A, B, I) \rightarrow H C_{n-1}(A, B, I) .
$$


Using the Loday-Quillen Theorem, Ogle and I have proven in $[\mathbf{O W}]$ that when $\mathbb{Q} \subset A$ and $B=A / J$ then indeed

$$
\nu: K_{n}(A, B, I) \cong H C_{n-1}(A, B, I) .
$$

Third of all, consider the groups $N K_{n}(A)$, which are the cokernels of $K_{n}(A) \rightarrow$ $K_{n}(A[t])$. We know that $K V_{n}(A)=K V_{n}(A[t])$ and that when $\mathbb{Q} \subset A$ the groups $N K_{n}(A)$ are $\mathbb{Q}$-vector spaces. When $\mathbb{Q} \subset A$ we also know (e.g. by [Kass] or 3.2 below) that $H P_{n}(A)=H P_{n}(A[t])$. It follows that we have a diagram of $\mathbb{Q}$-vector spaces, in what should be self-evident notation:

$$
\begin{gathered}
\operatorname{nil} N K_{n}(A) \cong N K_{n}(A) \\
N \nu \downarrow \\
N H C_{n-1}(A) \cong N H C_{n}^{-}(A)
\end{gathered}
$$

I do not know how often the map $N \nu \cong N \gamma$ is an injection. It clearly is not always an isomorphism. For example, if $A$ is a field of characteristic zero then $N K_{n}(A)=0$ for all $n$, but $N H C_{0}(A)=A[t] / A$ is not zero. In fact, by [Kass, 4.3]

$$
N H C_{n}(A) \cong \Omega_{A}^{n} \otimes t \mathbb{Q}[t]
$$

can be nonzero for all $n$, depending on the transcendence degree of $A$.

I originally constructed the maps $\mathrm{ch}$ and $\nu$ in March 1985 during the seminar [IAS]. I owe C. Kassel a debt of thanks for his encouragement. Subsequently, I discovered that ch was implicit in $[\mathbf{K 2}]$ and that Theorem $\mathrm{C}$ could be interpreted as a form of Theorem 7 of $[\mathbf{C K}]$, where nil $K$ is a case of $K^{\text {rel }}$. Since my viewpoint is much more elementary, and since this work motivated [OW], I hope the reader does not mind the overlap. I have tried to backtrack and annotate this paper with literature references in partial atonement.

1. Cyclic modules and their homology. In this section we recall some elementary facts about cyclic modules, establishing notation. It is difficult to give proper credit for these observations; I learned much of this material in the seminar [IAS], and from various conversations. I have tried to cite the literature after the fact, and apologize for any omissions. The main references are $[\mathbf{C}, \mathbf{K}, \mathbf{K} \mathbf{1}$, Bur, Bur 1, Gl and G].

Cyclic modules and their homology are generalizations of simplicial modules and their homotopy. For the sake of uniform notation let us agree that if $L$ is a simplicial $k$-module, then $L$. will denote the chain complex with differential $b=$ $\sum(-1)^{i} d_{i}: L_{n} \rightarrow L_{n-1}$, and $H H_{*}(L)$ will denote the homology of $L$. It is well known that $\pi_{*}(L)=H H_{*}(L)$ [May].

We shall be particularly interested in the simplicial modules $\operatorname{Cyc}(A ; M)$ attached to a $k$-algebra $A$ and an $A$-bimodule $M$. Writing $\otimes$ for $\otimes_{k}$ and $A^{\otimes n}$ for $A \otimes \cdots \otimes A$ ( $n$ times), we have $\mathrm{Cyc}_{n}(A ; M)=M \otimes A^{\otimes n}$. We have

$$
\begin{gathered}
s_{i}\left(m \otimes a_{1} \otimes \cdots \otimes a_{n}\right)=m \otimes \cdots \otimes a_{i} \otimes 1 \otimes a_{i+1} \otimes \cdots, \\
d_{i}\left(m \otimes a_{1} \otimes \cdots \otimes a_{n}\right)= \begin{cases}m a_{1} \otimes \cdots \otimes a_{n} & \text { if } i=0, \\
m \otimes \cdots \otimes a_{i} a_{i+1} \otimes \cdots & \text { if } 0<i<n, \\
a_{n} m \otimes a_{1} \otimes \cdots \otimes a_{n-1} & \text { if } i=n .\end{cases}
\end{gathered}
$$


The groups $H H_{*}(\operatorname{Cyc}(A ; M))$ are usually denoted $H_{*}(A ; M)$ or $H_{*}^{k}(A ; M)$. They are the Hochschild homology groups of $A$ with coefficients in $M$. (See [CE, Mac].)

DEFINITION 1.1 [C, Bur, G1]. A simplicial object $L$ in some category is called a cyclic object if each $L_{n}$ is given an automorphism $t$ of order $n+1$ satisfying the following combinatorial identities:

$$
d_{i} t=\left\{\begin{array}{ll}
d_{n} & \text { if } i=0, \\
t d_{i-1} & \text { if not, }
\end{array} \quad s_{i} t= \begin{cases}t^{2} s_{n} & \text { if } i=0, \\
t s_{i-1} & \text { if not. }\end{cases}\right.
$$

(The relations for $d_{0} t$ and $s_{0} t$ are redundant.)

The homology modules $H C_{*}(L), H P_{*}(L)$ and $H C_{*}^{-}(L)$ associated to a cyclic module $L$ were introduced in $[\mathbf{C}, \mathbf{L Q}, \mathbf{B u r}, \mathbf{G}$ and $\mathbf{G} \mathbf{1}]$. We shall recapitulate their construction shortly. First, however, we shall give some examples and derive some easy facts about them based on maps of cyclic modules.

ExAmPle 1.2.1 [C; G1, II.1]. $\operatorname{Cyc}(A ; A)$ becomes a cyclic $k$-module once we set

$$
t\left(a_{0} \otimes \cdots \otimes a_{n}\right)=a_{n} \otimes a_{0} \otimes \cdots \otimes a_{n-1} .
$$

Because it is so common, we shall write $H H_{*}(A)$ for the more awkward $H_{*}(A ; A)$ or $H H_{*}(\operatorname{Cyc}(A ; A))$. Similarly, we shall write $H C_{*}(A)$ for $H C_{*}(\operatorname{Cyc}(A ; A))$, and so on.

EXAMPLE 1.2.2. Let $M$ be a right $k G$-module and let ${ }_{\varepsilon} M$ denote the $k G$ bimodule whose underlying right module is $M$, but which is a trivial left $k G$-module, i.e., $g m=m$ for all $g$ in $G$. If we define $t$ by the formula

$$
t\left(m \otimes g_{1} \otimes \cdots \otimes g_{n}\right)=\left(m g_{1} \cdots g_{n}\right) \otimes\left(g_{1} \cdots g_{n}\right)^{-1} \otimes g_{1} \otimes \cdots \otimes g_{n-1}
$$

then $\operatorname{Cyc}\left(k G ;{ }_{\varepsilon} M\right)$ becomes a cyclic $k$-module. Again for simplicity, we shall write $H H_{*}(G ; M)$ for $H H_{*}\left(\operatorname{Cyc}\left(k G ;{ }_{\varepsilon} M\right)\right), H C_{*}(G ; M)$ for $H C_{*}\left(\operatorname{Cyc}\left(k G ;{ }_{\varepsilon} M\right)\right)$, and so on (cf. $[\mathbf{K}])$.

The notation in 1.2.2 is motivated by the classical fact that

$$
H H_{*}(G ; M) \equiv H_{*}^{k}\left(k G ;{ }_{\varepsilon} M\right)=H_{*}(G ; M)
$$

(see [CE, X.2.1; Mac, p. 291]). Here $H_{*}(G ; M)$ denotes the Eilenberg-Mac Lane homology of $G$ with coefficients in the $G$-module $M$.

EXAMPLE 1.3.1 (MORITA INVARIANCE). The inclusion of $A$ in the matrix ring $M_{r} A$ induces a cyclic map from $\operatorname{Cyc}(A ; A)$ to $\operatorname{Cyc}\left(M_{r} A ; M_{r} A\right)$. There is a cyclic map going in the other direction:

$$
\text { trace: } \begin{aligned}
\operatorname{Cyc}_{n}\left(M_{r} A ; M_{r} A\right) \rightarrow \operatorname{Cyc}_{n}(A ; A) \\
g^{0} \otimes \cdots \otimes g^{n} \mapsto \sum g_{i_{0} i_{1}}^{0} \otimes g_{i_{1} i_{2}}^{1} \otimes \cdots \otimes g_{i_{n} i_{0}}^{n} .
\end{aligned}
$$

A priori, this shows that $\operatorname{Cyc}(A ; A)$ is a direct summand of $\operatorname{Cyc}\left(M_{r} A ; M_{r} A\right)$ in the category of cyclic $k$-modules.

In fact, we know that "trace" induces isomorphisms in Hochschild homology [DI, 3.7], as well as in $H C$ [LQ, 1.7], $H P$ and $H C^{-}$[G, 1.3.8].

EXAMPLE 1.3.2. It is a classical fact that $H_{*}(G ; k)$ is a direct summand of $H H_{*}(k G)$. For example, this is implicit in [CE, X.2.1] and [Mac, p. 291], because $k$ is a summand of the right $G$-module ${ }_{\chi}(k G)$.

In fact, $\operatorname{Cyc}\left(k G ;{ }_{\varepsilon} k\right)$ is naturally a direct summand of $\operatorname{Cyc}(k G ; k G)$ in the category of cyclic $k$-modules. 
Consequently, if $H X$ denotes any of the homology theories $H H, H C, H P$ or $H C^{-}$then $H X_{*}(G ; k)$ is naturally a direct summand of $H X_{*}(k G)$. The classical fact cited above follows from this and (1.2.3).

Although this observation is new, the proof is not. It is explicit in [D], and dates back to the 1950's. Variations of this observation may be found in [K and Bur]. One defines maps for each $n$,

$$
\begin{aligned}
& \mathrm{Cyc}_{n}\left(k G ;{ }_{\varepsilon} k\right) \stackrel{\iota}{\rightarrow} \mathrm{Cyc}_{n}(k G ; k G) \stackrel{\pi}{\rightarrow} \mathrm{Cyc}_{n}\left(k G ;{ }_{\varepsilon} k\right), \\
& 1 \otimes g_{1} \otimes \cdots \otimes g_{n} \mapsto\left(g_{1} \cdots g_{n}\right)^{-1} \otimes g_{1} \otimes \cdots \otimes g_{n}, \\
& g_{0} \otimes g_{1} \otimes \cdots \otimes g_{n} \mapsto \begin{cases}1 \otimes g_{1} \otimes \cdots \otimes g_{n} & \text { if } g_{0}=\left(g_{1} \cdots g_{n}\right)^{-1}, \\
0 & \text { if not. }\end{cases}
\end{aligned}
$$

Next, one verifies that $\iota$ and $\pi$ are cyclic maps, and that $\pi \iota$ is the identity. The result follows.

Of the various homology groups associated to a cyclic module $L, H C_{*}(L)$ is best known. As in [C, G1 or LQ], there is a first quadrant double complex $L_{p q}^{+}(p \geq 0$ and $q \geq 0)$ such that $H C_{n}(L)=H_{n}\left(\operatorname{tot}\left(L_{. .}^{+}\right)\right)$. The even columns of $L_{. .}^{+}$are each the chain complex $(L ., b)$; the odd columns are each the chain complex $\left(L .,-b^{\prime}\right)$, where $b^{\prime}: L_{q} \rightarrow L_{q-1}$ is the map $b^{\prime}=\sum_{i \neq q}(-1)^{i} d_{i}$. The horizontal maps are either $1-T$ or $N=1+T+\cdots+T^{q}, T=(-1)^{q} t$, depending on the parity of the columns.

The same prescription gives an upper half-plane double complex $L . .(q \geq 0)$, and a second quadrant double complex $L_{. \cdot}^{-}(p \leq 0, q \geq 0)$. $H P_{*}(L)$ is defined to be the homology of $\operatorname{tot}\left(L_{. .}\right)$and $H C_{*}^{-}(L)$ is defined to be the homology of tot $\left(L_{. .}^{-}\right)$. Note that the total complex of $L .$. is given by

$$
\operatorname{tot}(L . .)_{n}=\prod_{p+q=n} L_{p q} .
$$

It is easy to compute $H P$ in terms of $H C$. Since the columns of $L .$. are periodic, we can write $L . .=\lim _{\longleftarrow} L_{. .}^{+}$; from this it follows that there is an exact sequence

$$
0 \rightarrow \underline{\lim }^{1} H C_{n+2 p}(L) \rightarrow H P_{n}(L) \rightarrow \lim _{\leftarrow} H C_{n+2 p}(L) \rightarrow 0 .
$$

The $H C^{-}$groups bear the same relation to $H P$ and $H C$ that the $H H$ groups bear to $H C$. That is, we can copy the proof of $[\mathbf{L Q}, 1.6]$, mutatis mutandis, to prove the following result (see [C or $\mathbf{G}, \mathbf{I} .3 .1]$ ).

PROPOSITION 1.5 (CONNES-GYSIN SEQUENCES). If $L$ is a cyclic $k$-module, there is a map of long exact sequences:

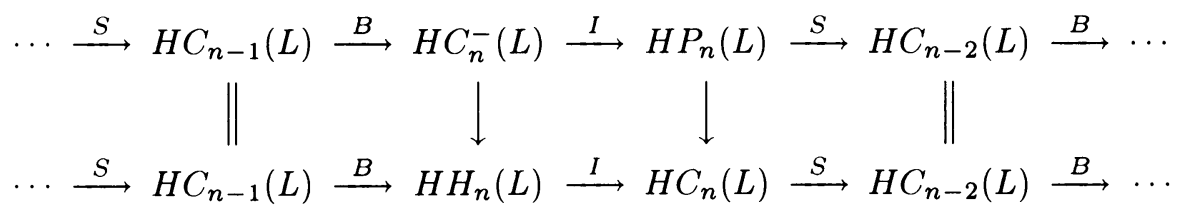

COROLlaRY 1.5.1. If $n \leq 0$ then

$$
H C_{n}^{-}(L)=H P_{n}(L),
$$

and $H C_{1}^{-}(L) \rightarrow H P_{1}(L)$ is onto. 
EXAMPLE 1.5.2. When $L=\operatorname{Cyc}(k ; k)$, we see from [LQ] that

$$
H H_{n}(k)=\left\{\begin{array}{ll}
k & \text { if } n=0, \\
0 & \text { if not, }
\end{array} \quad H C_{n}(k)= \begin{cases}k & \text { if } n \text { is even, } n \geq 0, \\
0 & \text { if not. }\end{cases}\right.
$$

It follows easily that

$$
H P_{n}(k)=\left\{\begin{array}{ll}
k & \text { if } n \text { is even, } \\
0 & \text { if } n \text { is odd, }
\end{array} \quad H C_{n}^{-}(k)= \begin{cases}k & \text { if } n \text { is even, } n \leq 0, \\
0 & \text { if not. }\end{cases}\right.
$$

The Connes-Gysin sequences break up because $B=0$, and the vertical maps in 1.5 are isomorphisms for $n \geq 0$.

EXERCISE 1.6. Let ${ }_{\varepsilon} k G$ denote the right $k G$-module $k G$, made into a bimodule with $G$ acting trivially on the left. By 1.2 .3 we know that

$$
H H_{n}(G ; k G)=H_{n}(G ; k G)= \begin{cases}k & \text { if } n=0 \\ 0 & \text { if not. }\end{cases}
$$

Show that the double complex Cyc.. $\left(k G ;{ }_{\varepsilon} k G\right)$ is a free $G$-resolution of the chain complex $K .: \cdots \leftarrow k \leftarrow 0 \leftarrow k \leftarrow 0 \leftarrow k \leftarrow \cdots$. (Here at last we allow $G$ to act on the left of ${ }_{\varepsilon} k G$.) Conclude from this that

$$
H P_{n}(G ; k G)= \begin{cases}k & \text { if } n \text { is even, } \\ 0 & \text { if } n \text { is odd. }\end{cases}
$$

Finally, compare this exercise with the discussion on p. 382 of $[\mathbf{K}]$.

It is time for our main calculation.

ThEOREM 1.7 (KAROUBI $[\mathrm{K}]$ ). We have

$$
\begin{aligned}
& H H_{n}(G ; k) \cong H_{n}(G ; k), \\
& H C_{n}(G ; k) \cong H_{n}(G ; k) \oplus H_{n-2}(G ; k) \oplus \cdots, \\
& H P_{n}(G ; k) \cong \prod_{p=-\infty}^{+\infty} H_{n+2 p}(G ; k), \\
& H C_{n}^{-}(G ; k) \cong \prod_{p=0}^{\infty} H_{n+2 p}(G ; k) .
\end{aligned}
$$

The Connes-Gysin sequences break up into short exact sequences, the $B$ maps being zero. Finally, the maps $H C_{n}^{-} \rightarrow H H_{n}$ and $H P_{n} \rightarrow H C_{n}$ are the natural surjections given by the above formulas.

FIRST PROOF. The $H H$ calculation is from 1.2.3, and the $H C$ calculation was done by Karoubi on p. 382 of $[\mathbf{K}]$. The $H P$ calculation now follows from (1.4), and the $\mathrm{HC}^{-}$calculation from 1.5 .

SECOND PROOF. This is an extension of Karoubi's calculation in $[\mathbf{K}]$. We adopt the notation of exercise 1.6 above; since Cyc.. $\left(k G ;{ }_{\varepsilon} k G\right)$ is a free $G$-resolution of $K$. , and since Сyc.. $\left(k G ;{ }_{\varepsilon} k\right)=G \backslash$ Суc.. $\left(k G ;{ }_{\varepsilon} k G\right)$, a hyperhomology calculation yields

$$
\begin{aligned}
H P_{n}(G ; k) & =\text { hyper } H_{n}(G ; K .) \\
& =\lim _{\leftarrow} \text { hyper } H_{n}(G ; k \leftarrow 0 \leftarrow \cdots \leftarrow k \leftarrow \cdots) \\
& =\prod H_{n+2 p}(G ; k) .
\end{aligned}
$$


In this calculation we used the easily checked fact that the system

$$
\left\{\text { hyper } H_{n}(G ; k \leftarrow \cdots)\right\}
$$

satisfies the Mittag-Leffler condition, so that $\lim ^{1}=0$. The same formal calculation goes through for $H C$ and $H C^{-}$.

REMARK 1.8. Although there is no canonical splitting of the projection from $H C_{n}^{-} \cong \prod H_{n+2 p}(G ; k)$ onto $H H_{n} \cong H_{n}(G ; k)$, it is possible to select splittings which are natural in $G$. In fact, we can find splittings of the underlying chain map

$$
\operatorname{tot}_{n}=\operatorname{tot}\left(\mathrm{Cyc}_{. .}^{-}(k G ; k)\right)_{n} \rightarrow C y c_{n}(k G ; k) .
$$

Here we have written $k$ for the bimodule ${ }_{\varepsilon} k$. This is essentially the content of Lemma II.3.2 in $[\mathbf{G}]$ and uses acyclic models. First choose the canonical lift of $k=\mathrm{Cyc}_{0}(k G ; k)$. Next, let $Z$ be infinite cyclic on $z$ and choose a lifting of $1 \otimes z \in$ $\mathrm{Cyc}_{1}(k Z ; k)$ to a cycle; this is possible by 1.7 because $H C_{1}^{-} \cong H H_{1} \cong k$. Use this to find natural liftings of all $1 \otimes g$, hence a natural splitting $\mathrm{Cyc}_{1}(k G ; k) \rightarrow$ tot $_{1}$. Similarly, $\operatorname{Cyc}_{p}(k G ; k)$ is a free $k$-module for $p \geq 2$, and is modeled by the case in which $G$ is a free group on $p$ generators. In this case we know by 1.7 that both complexes in 1.8.1 are exact at $p$, so there is no difficulty in inductively finding a natural map $\mathrm{Cyc}_{p}(k G ; k) \rightarrow \operatorname{tot}_{p}$ compatible with the differentials.

2. Proof of Theorem A. In this section, we will take $k=\mathbb{Z}$, let $A$ be a ring with unit, and fix $G=\operatorname{GL}(A)$. For $n \geq 1$, let $h$ denote the Hurewicz map:

$$
K_{n}(A)=\pi_{n} B \mathrm{GL}^{+}(A) \rightarrow H_{n}\left(B \mathrm{GL}^{+}(A) ; \mathbb{Z}\right) \cong H_{n}(B G ; \mathbb{Z}) \cong H_{n}(G ; \mathbb{Z}) .
$$

Let $M_{i}(A)$ denote the ring of $i \times i$ matrices over $A, M A=\bigcup M_{i}(A)$, and let $\mathbb{Z} G \rightarrow M A$ denote the ring map induced from the inclusions of $\mathrm{GL}_{i}(A)$ into $M_{i}(A)$. Finally, choose a splitting $s$ of $H C_{*}^{-}(G ; k) \rightarrow H H_{*}(G ; k)$ using 1.7 and 1.8. There is a commutative diagram (for $n \geq 1$ ):

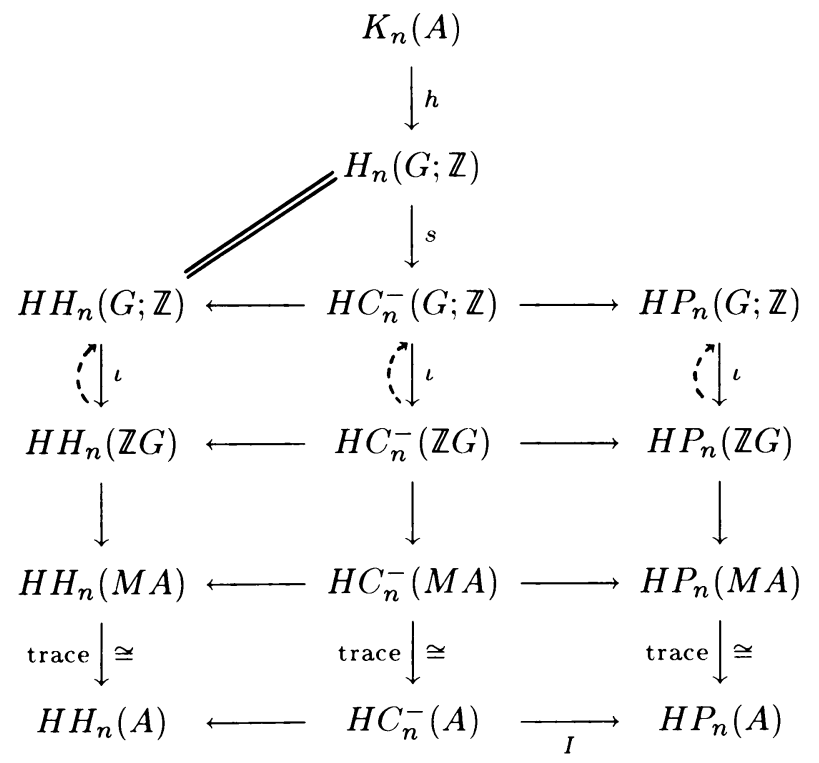


The split injections $\iota$ and the trace maps are defined in 1.3. The composite $K_{n}(A) \rightarrow H H_{n}(A)$ is the Dennis trace map as defined in [D], and the composite $K_{n}(A) \rightarrow H C_{n}^{-}(A)$ is Goodwillie's map $\gamma$ of [G]. I claim that the composite $I \gamma: K_{n}(A) \rightarrow H P_{n}(A)$ is due to Karoubi.

Proposition 2.2. The composite of the map $K_{n}(A) \rightarrow H P_{n}(A)$ in (2.1) and the projection $H P_{n}(A) \rightarrow \lim _{\longleftarrow} H C_{n+2 p}(A)$ in (1.4) is the Chern class map defined by Karoubi in [K1].

PROOF. Reinterpreting the construction in [K1] using

$$
\lim _{\leftarrow} H C_{n+2 p}(G ; \mathbb{Z}) \cong H P_{n}(G ; \mathbb{Z}) \cong \prod_{p=-\infty}^{+\infty} H_{n+2 p}(G ; \mathbb{Z}),
$$

the Chern class of $[\mathbf{K 1}]$ is the composite

$$
\begin{aligned}
K_{n}(A) & \stackrel{h}{\rightarrow} H_{n}(G ; \mathbb{Z}) \stackrel{\kappa}{\rightarrow} \prod_{p=-\infty}^{+\infty} H_{n+2 p}(G ; \mathbb{Z}) \\
\cong H P_{n}(G ; \mathbb{Z}) & \rightarrow H P_{n}(A) \rightarrow \lim _{\leftarrow} H C_{n+2 p}(A),
\end{aligned}
$$

where the map $H P_{n}(G ; \mathbb{Z}) \rightarrow H P_{n}(A)$ is from diagram (2.1). The key map here is $\kappa$, and it can be taken to be

$$
H_{n}(G ; \mathbb{Z}) \stackrel{s}{\rightarrow} \prod_{p=0}^{\infty} H_{n+2 p}(G ; \mathbb{Z}) \cong H C_{n}^{-}(G ; \mathbb{Z}) \rightarrow H P_{n}(G ; \mathbb{Z})
$$

With this choice, the Chern class map becomes the map in (2.1).

3. Calculations. The best way to understand the theory in the previous sections (and next) is to perform a few calculations. Throughout this section, we compute over $k=\mathbb{Z}$.

EXAMPLE 3.1 . Let $R=\mathbb{Z}[t]$, so that $\Omega_{R} \cong R$ on $d t$ and $\Omega_{R} / d R \cong \bigoplus(\mathbb{Z} / j)$ on generators $t^{j-1} d t$. From $[\mathbf{L Q}, 2.6]$ we see that

$$
\left.H C_{n}(\mathbb{Z}[t])\right] \begin{cases}\mathbb{Z}[t] & \text { if } n=0, \\ \mathbb{Z} & \text { if } n \text { is even, } n \geq 2, \\ \bigoplus(\mathbb{Z} / j) & \text { if } n \text { is odd, } n \geq 1, \\ 0 & \text { if } n<0 .\end{cases}
$$

Since $H H_{n}(\mathbb{Z}[t])=0$ for $n \geq 2$, it follows from 1.5 that

$$
H P_{n}(\mathbb{Z}[t])= \begin{cases}\mathbb{Z} & \text { if } n \text { is even, } \\ \bigoplus(\mathbb{Z} / j) & \text { if } n \text { is odd. }\end{cases}
$$

In particular, note that $H P_{*}(\mathbb{Z}[t]) \neq H P_{*}(\mathbb{Z})$.

In contrast, we offer the more pleasing

LEMMA 3.2. If $\mathbb{Q} \subset A$ then $H P_{*}(A[t]) \cong H P_{*}(A)$.

ProOF. By [Kass, 4.3] with $B=\mathbb{Q}[t]$, we have

$$
H C_{n}(A[t])=H C_{n}(A) \oplus H H_{n}(A) \otimes t \mathbb{Q}[t]
$$


as a comodule over $\mathbb{Z}[S]$. That is, $S=0$ on the second term. The result follows from (1.4).

When $I$ is an ideal we shall write $H X_{*}(A, I)$ for $H X_{*}(L)$, where the cyclic module $L$ is the kernel of $\operatorname{Cyc}(A ; A) \rightarrow \operatorname{Cyc}(A / I ; A / I)$. Thus there are exact sequences

$$
\cdots H X_{n+1}(A / I) \rightarrow H X_{n}(A, I) \rightarrow H X_{n}(A) \rightarrow H X_{n}(A / I) \cdots
$$

The easiest case of this is when $A=\mathbb{Q}[\varepsilon], I=\mathbb{Q} \varepsilon$. From [LQ, (4.3)] we obtain

EXAMPLE 3.3.

$$
\begin{aligned}
& H H_{n}(\mathbb{Q}[\varepsilon], \mathbb{Q} \varepsilon)= \begin{cases}\mathbb{Q} & \text { if } n \geq 0, \\
0 & \text { if } n<0,\end{cases} \\
& H C_{n}(\mathbb{Q}[\varepsilon], \mathbb{Q} \varepsilon)= \begin{cases}\mathbb{Q} & \text { if } n \text { is even, } n \geq 0, \\
0 & \text { if not. }\end{cases}
\end{aligned}
$$

Every map $S$ is 0 , and the Connes-Gysin sequence breaks up. From this and (1.4), we see that

$$
\begin{aligned}
& H P_{n}(\mathbb{Q}[\varepsilon], \mathbb{Q} \varepsilon)=0 \\
& H C_{n}^{-}(\mathbb{Q}[\varepsilon], \mathbb{Q} \varepsilon)= \begin{cases}\mathbb{Q} & \text { if } n \text { is odd, } n \geq 1, \\
0 & \text { if not. }\end{cases}
\end{aligned}
$$

Goodwillie proved in $[\mathbf{G}]$ that there are isomorphisms

$$
K_{n}(\mathbb{Q}[\varepsilon], \mathbb{Q} \varepsilon) \underset{\gamma}{\cong} H C_{n}^{-}(\mathbb{Q}[\varepsilon], \mathbb{Q} \varepsilon) \underset{B}{\stackrel{\cong}{\leftrightarrows}} H C_{n-1}(\mathbb{Q}[\varepsilon], \mathbb{Q} \varepsilon) .
$$

In fact, by [Ogle, §4] we know for every odd $n$ that Loday's double bracket symbol $\langle\langle\varepsilon, \varepsilon, \ldots, \varepsilon\rangle\rangle$ in $K_{n}(\mathbb{Q}[\varepsilon], \mathbb{Q})$ is nonzero, because it maps nontrivially into $H H_{n}(\mathbb{Q}[\varepsilon])$. (This symbol is defined in [L and Ogle].)

EXAMPLE 3.4. When $A=\mathbb{Z}[\varepsilon]$ we encounter more delicate calculations. It is easy to directly compute that

$$
H H_{n}(\mathbb{Z}[\varepsilon], \mathbb{Z} \varepsilon)= \begin{cases}\mathbb{Z} & \text { if } n \text { is even, } n \geq 0 \\ \mathbb{Z} \oplus \mathbb{Z} / 2 & \text { if } n \text { is odd, } n \geq 1 \\ 0 & \text { if } n<0 .\end{cases}
$$

The element $\varepsilon \otimes \cdots \otimes \varepsilon$ of $L_{n}$ is a cycle. If $n$ is even it generates the $\mathbb{Z}$; if $n$ is odd it generates the $\mathbb{Z} / 2$. The $\mathbb{Z}$ for $n$ odd is generated by $1 \otimes \varepsilon \otimes \cdots \otimes \varepsilon$. From [LQ, (4.3) or Ogle, 2.1] we get

$$
H C_{n}(\mathbb{Z}[\varepsilon], \mathbb{Z} \varepsilon)= \begin{cases}\mathbb{Z} & \text { if } n \text { is even, } n \geq 0, \\ \prod_{\substack{m=1 \\ m \text { odd } \\ 0}}^{n}(\mathbb{Z} / m \oplus \mathbb{Z} / 2) & \text { if } n \text { is odd, } n \geq 1, \\ \text { if } n<0 .\end{cases}
$$

From [Ogle, 2.1], we see that the map $S: H C_{n} \rightarrow H C_{n-2}$ is zero if $n$ is even, and the evident projection if $n$ is odd. Thus

$$
H P_{n}(\mathbb{Z}[\varepsilon], \mathbb{Z} \varepsilon)= \begin{cases}0 & \text { if } n \text { is even, } \\ \prod_{\substack{m=1 \\ m}}^{\infty}(\mathbb{Z} / m \oplus \mathbb{Z} / 2) & \text { if } n \text { is odd }\end{cases}
$$


From this, or by performing a similar calculation, we get

$$
H C_{n}^{-}(\mathbb{Z}[\varepsilon], \mathbb{Z} \varepsilon)= \begin{cases}0 & \text { if } n \text { is even, } \\ (\mathbb{Z} \oplus \mathbb{Z} / 2) \oplus \prod_{\substack{m>n \\ m \text { odd }}}^{\infty}(\mathbb{Z} / m \oplus \mathbb{Z} / 2) & \text { if } n \text { is odd, } n \geq 1, \\ \prod_{\substack{m=1 \\ m \text { odd }}}^{\infty}(\mathbb{Z} / m \oplus \mathbb{Z} / 2) & \text { if } n \text { is odd, } n<0 .\end{cases}
$$

Now it is well known that $K_{1}(\mathbb{Z}[\varepsilon], \mathbb{Z} \varepsilon)=(1+\mathbb{Z} \varepsilon)^{*} \cong \mathbb{Z}$ and that the Dennis trace map sends $u$ to $u^{-1} \otimes u$. In particular, $u=1+\varepsilon$ maps to the cycle $(1-\varepsilon) \otimes(1+\varepsilon) \sim$ $1 \otimes \varepsilon-\varepsilon \otimes \varepsilon$ which under the given isomorphism $H H_{1} \cong \mathbb{Z} \oplus \mathbb{Z} / 2$ corresponds to $(1,1)$. In $H C_{1} \cong \mathbb{Z} / 2$ the cycle $1 \otimes \varepsilon$ is zero but $\varepsilon \otimes \varepsilon$ is nonzero. We record the following consequence:

OBSERVATION 3.4.1. Goodwillie's map $\gamma: K_{1}(\mathbb{Z}[\varepsilon], \mathbb{Z} \varepsilon) \rightarrow H C_{1}^{-}(\mathbb{Z}[\varepsilon], \mathbb{Z} \varepsilon)$ is injective, because the Dennis trace map is. In addition, Karoubi's Chern class is nonzero, because

$$
\mathbb{Z} \cong K_{1}(\mathbb{Z}[\varepsilon], \mathbb{Z} \varepsilon) \stackrel{\mathrm{ch}}{\longrightarrow} H P_{1}(\mathbb{Z}[\varepsilon], \mathbb{Z} \varepsilon) \rightarrow H C_{1}(\mathbb{Z}[\varepsilon], \mathbb{Z} \varepsilon) \cong \mathbb{Z} / 2
$$

is nonzero.

4. The space level. In this section, we shall realize the maps in (2.1) at the topological level. We shall start with the map $h$.

If $X$ is a simplicial set, let $\mathbb{Z} X$ denote the simplicial abelian group which in degree $n$ is the free abelian group on the set $X_{n}$. Almost by definition the homology of $X, H_{*}(X ; \mathbb{Z})$, is the homotopy $\pi_{*}(\mathbb{Z} X)=H H_{*}(\mathbb{Z} X)$. There is a simplicial map $X \rightarrow \mathbb{Z} X$ (sending $x$ to $x$ ), and it is classical [May] that the Hurewicz map is just $\pi_{*}(X) \rightarrow \pi_{*}(\mathbb{Z} X)=H_{*}(X ; \mathbb{Z})$.

Now let $G$ be a group, and let $N$ be the nerve of $G$, i.e., the simplicial set whose realization $|N|$ is $B G$. Almost by definition, the simplicial abelian group $\mathbb{Z} N$ is $\operatorname{Cyc}(\mathbb{Z} G ; \mathbb{Z})$. Specializing even further, let $G=\operatorname{GL}(A)$. Since addition makes the realization of any simplicial abelian group into an $H$-space, it follows that there is a map $B \mathrm{GL}^{+}(A) \rightarrow|\mathrm{Cyc}(\mathbb{Z} G ; \mathbb{Z})|$, unique up to homotopy, such that

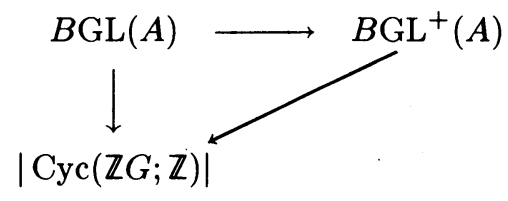

is homotopy commutative. From these remarks and the construction of $h$, we obtain

LeMmA 4.1. The map $B \mathrm{GL}^{+}(A) \rightarrow|\operatorname{Cyc}(\mathbb{Z} G ; \mathbb{Z})|$ induces the map $h: K_{n}(A)$ $\rightarrow H_{n}(\mathrm{GL}(A) ; \mathbb{Z})$.

Next, we turn to the maps in the lower part of (2.1). These are induced from maps of chain complexes of abelian groups. In order to obtain topological spaces, we use the Dold-Kan theorem, which states that the category of simplicial abelian groups is equivalent to the category $\mathrm{Ch}$ of chain complexes $C$. with $C_{n}=0$ for $n<$ 0 . Under this correspondence, homotopy corresponds to homology. Unfortunately, the chain complexes involved are not in $\mathrm{Ch}$, so we need to truncate. 
If $C$. is a chain complex, let $0 \backslash C$. denote the subcomplex

$$
(0 \backslash C)_{n}= \begin{cases}0 & \text { if } n<0 \\ \operatorname{ker}\left(C_{0} \rightarrow C_{-1}\right) & \text { if } n=0 \\ C_{n} & \text { if } n>0\end{cases}
$$

Then $0 \backslash C$. is in $\mathbf{C h}$ and $H_{n}(0 \backslash C)=.H_{n}(C$.$) for n \geq 0$. In fact, $0 \backslash$ is the reflection into $\mathbf{C h}$; if $P$. is in $\mathrm{Ch}$ then

$$
\operatorname{Hom}(P ., C .)=\operatorname{Hom}(P ., 0 \backslash C .)
$$

If we write $D K$ for the Dold-Kan equivalence from $\mathrm{Ch}$ to simplicial abelian groups, and $|L|$ for the realization of $L$, we have

$$
\left[\begin{array}{c}
\text { Chain } \\
\text { complexes }
\end{array}\right] \stackrel{0 \backslash}{\longrightarrow} \mathrm{Ch} \stackrel{D K}{\longrightarrow}\left[\begin{array}{c}
\text { Simplicial } \\
\text { abelian groups }
\end{array}\right] \stackrel{|\cdot|}{\longrightarrow} \text { Spaces. }
$$

To streamline notation, let us agree to write $X(C)$ for $|D K(0 \backslash C)|$. From the above remarks, we see that

$$
H_{n}(C)=\pi_{n} X(C) \text { for } n \geq 0
$$

In particular, $H C_{n}^{-}(A)$ and $H P_{n}(A)$ are the homotopy groups (for $n \geq 0$ ) of the spaces

$$
X\left(\operatorname{tot}\left(\mathrm{Cyc}_{.} .(A ; A)\right)\right) \quad \text { and } \quad X(\operatorname{tot}(\text { Сyc.. }(A ; A))) .
$$

Since all the maps in the bottom of (2.1) are induced in homology by chain maps, it follows that they may also be considered to be induced in homotopy by maps of spaces.

To get the map $s$ of (2.1), recall from 1.8 that $s$ is induced by a chain map (1.8.1). It follows that $s$ is induced in homotopy by a map of spaces $X($ Cyc. $(\mathbb{Z} G ; \mathbb{Z})) \rightarrow$ $X(\operatorname{tot}($ Cyc.. $(\mathbb{Z} G ; \mathbb{Z})))$, where $G=\operatorname{GL}(A)$. But there is a homotopy equivalence of simplicial abelian groups $\mathrm{Cyc}(\mathbb{Z} G ; \mathbb{Z}) \rightarrow D K(\mathrm{Cyc} .(\mathbb{Z} G ; \mathbb{Z}))$, so $s$ is induced by the map of spaces

$$
|\mathrm{Cyc}(\mathbb{Z} G ; \mathbb{Z})| \rightarrow X\left(\operatorname{tot}\left(\mathrm{Cyc} . .^{-}(\mathbb{Z} G ; \mathbb{Z})\right)\right) .
$$

Putting 4.1 and (4.2) together, we see that we have proven the following proposition. 
PROPOSITION 4.3. The diagram (2.1) arises from applying $\pi_{n}$ to the following diagram of spaces:

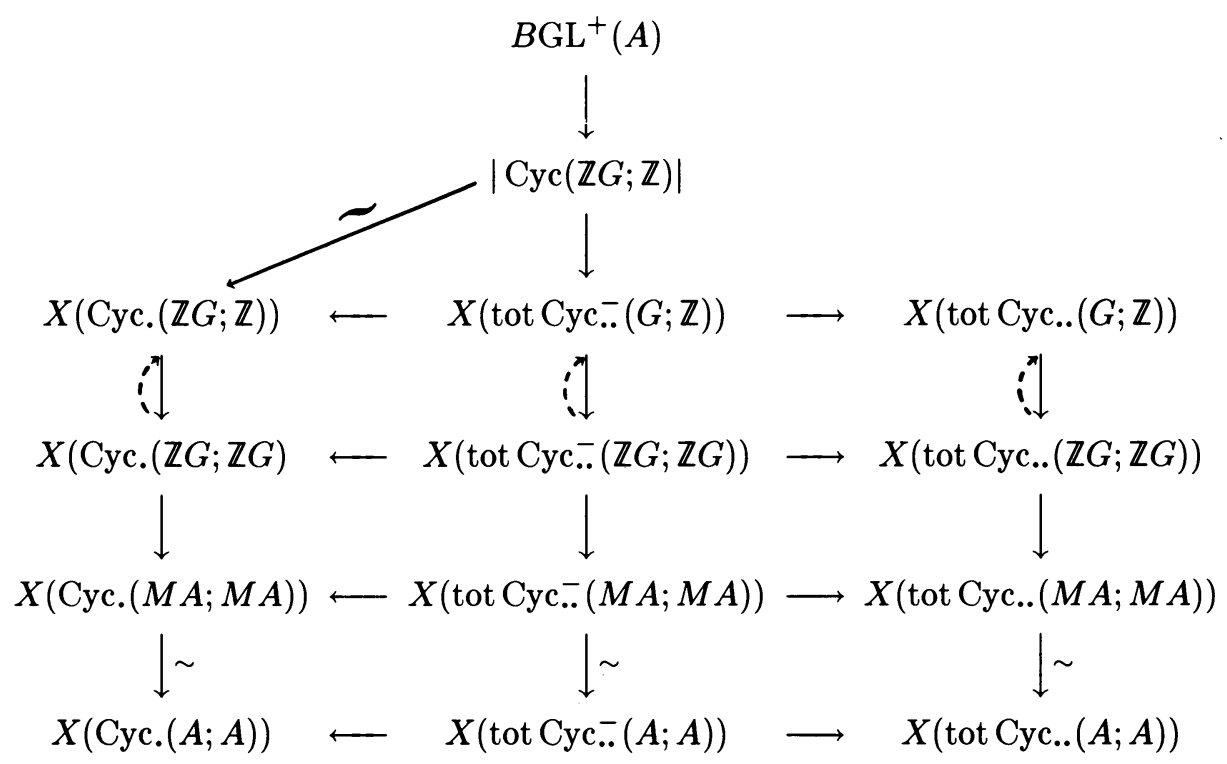

We shall conclude this section with a description of the Connes-Gysin sequence at the space level. Let $\Sigma$ denote suspension of chain complexes, so $H_{n}(\Sigma C)=$ $H_{n-1}(C)$. Given a cyclic group $L$, consider the diagram of chain complexes (whose rows are not exact in the middle):

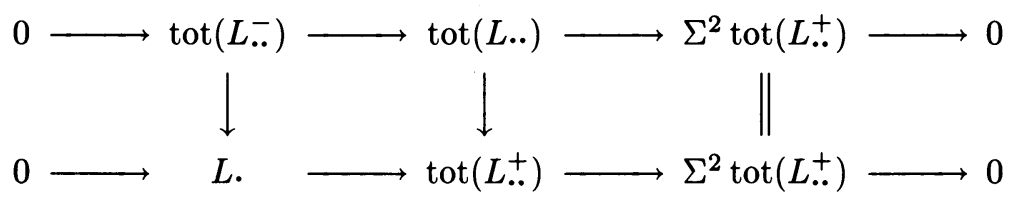

As in $[\mathbf{L Q}, 1.6]$, these induce the Connes-Gysin sequences of 1.5 when we apply homology. Since $X$ sends homology to homotopy, we have

PROPOSITION 4.4. The following diagram of topological spaces commutes, and the rows are homotopy fibrations:

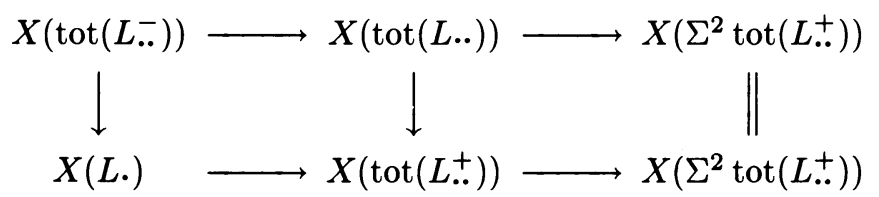

Moreover, the long exact sequences on homotopy are the Connes-Gysin sequences of 1.5 .

REMARK 4.4.1. If $C$ is in $\mathrm{Ch}$, then $\Omega X(\Sigma C) \simeq X(C)$. Thus $X(C)$ is an infinite loop space. Agreeing that $\Omega^{-1} X(C)$ denotes the connected space $X(\Sigma C)$, 
4.4 induces the map of homotopy fibrations

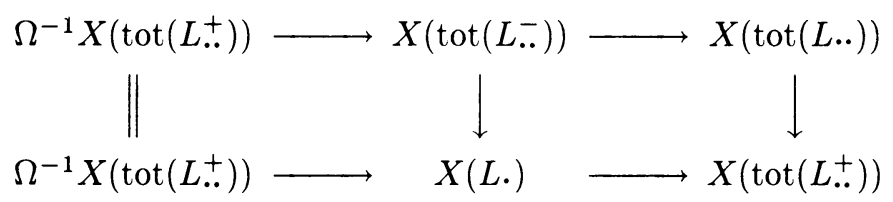

5. The maps ch and $\nu$. In this section, we shall factor Karoubi's Chern class map for $\mathbb{Q}$-algebras through $K V$-theory. Breaking $K$-theory up into the two pieces $K V$ and nil $K$, we will also induce a map (for $\mathbb{Q} \subset A$ ) $\nu: \operatorname{nil} K_{n}(A) \rightarrow H C_{n-1}(A)$ compatible with ch, proving Theorem $\mathrm{C}$.

Example 3.4 above shows that the assumption $\mathbb{Q} \subset A$ is necessary; $K V_{1}(\mathbb{Z}[\varepsilon], \mathbb{Z} \varepsilon)$ $=0$ holds, yet as observed in (3.4.1) the map $K_{1} \rightarrow H P_{1}$ is nonzero. In fact, the difficulty may be traced to the fact that $H P_{*}$ is not homotopy invariant unless we restrict to $\mathbb{Q}$-algebras. This problem is illustrated by Example 3.1 and Lemma 3.2.

We need to recall the construction of $K V$ from [And]. Given a ring $A$, let $\Delta A$ denote the simplicial ring with

$$
\begin{gathered}
\Delta_{n} A=A\left[t_{0}, \ldots, t_{n}\right] /\left(\Sigma t_{i}=1\right) \cong A\left[t_{1}, \ldots, t_{n}\right] \\
d_{i}\left(t_{i}\right)=0 \text { and } s_{i}\left(t_{i}\right)=t_{i}+t_{i+1} .
\end{gathered}
$$

If $i \neq j$ we have $d_{i}\left(t_{j}\right)=t_{j}$ or $t_{j-1}$ and $s_{i}\left(t_{j}\right)=t_{j}$ or $t_{j+1}$. Heuristically, $\Delta_{n} A$ is the ring of functions on the $n$-simplex, and the face and degeneracy maps are determined by geometry.

DEFINITIONS 5.2. Set $K(A)=B \mathrm{GL}^{+}(A)$ and $K V(A)=\left|B \mathrm{GL}^{+}(\Delta A)\right|(=$ the geometric realization of the simplicial space $K(\Delta A)$ ). Note that $K_{n}(A)=\pi_{n} K(A)$ and $K V_{n}(A)=\pi_{n} K V(A)$ for $n \geq 1$ [And]. Let nil $K(A)$ denote the homotopy fiber of $K(A) \rightarrow K V(A)$. For $n \geq 1$, we define

$$
\operatorname{nil} K_{n}(A)=\pi_{n} \operatorname{nil} K(A) \text {. }
$$

The long exact homotopy sequence for the fibration nil $K \rightarrow K \rightarrow K V$ is thus:

$$
\cdots \rightarrow \operatorname{nil} K_{2}(A) \rightarrow K_{2}(A) \rightarrow K V_{2}(A) \rightarrow \operatorname{nil} K_{1}(A) \rightarrow K_{1}(A) \rightarrow K V_{1}(A) \rightarrow 0
$$

REMARK 5.2.1. One usually defines $K V_{n}(A)=K_{n}(A)$ for $n \leq 0$. To be consistent, we would therefore set nil $K_{n}(A)=0$ for $n \leq 0$. Such a convention would allow us to extend Theorem $\mathrm{C}$ to all values of $n$. However, such an extension introduces extra technical complications, as in $[\mathbf{W}]$, so we shall forego discussing such an extension here.

REMARK 5.2.2. The above definition of nil $K_{n}(A)$ is different from that of [W1, 4.1], where I defined nil $K_{n}(A)$ to be the kernel of $K_{n}(A) \rightarrow K V_{n}(A)$. I will elaborate on this point in $\S 6$.

Now recall from $\S 4$ that there is a topological space $Y(A)=X(\operatorname{tot}$ Cyc.. $(A ; A))$, depending functorially on $A$, with the property that $\pi_{n} Y(A)=H P_{n}(A)$. By 4.3, we have the diagram

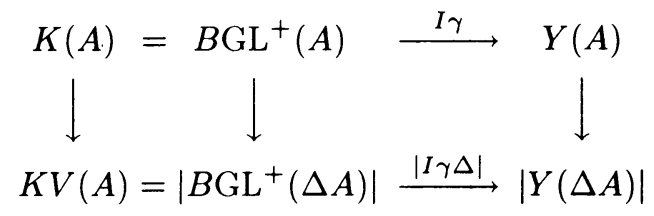


It can be seen from Examples 3.1 and 3.4 above that $Y(A) \rightarrow|Y(\Delta A)|$ is not a homotopy equivalence for $A=\mathbb{Z}[\varepsilon]$. However, we do have

PROPOSITION 5.3. If $\mathbb{Q} \subset A$ then $Y(A) \rightarrow|Y(\Delta A)|$ is a homotopy equivalence.

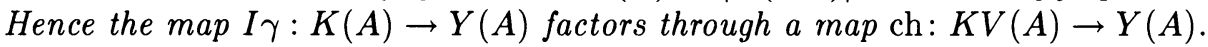

ProOF. By 3.2 above, $Y(A) \rightarrow Y\left(\Delta_{n} A\right)$ is a homotopy equivalence for each $n$. It is well known that this implies the result. (See $[\mathbf{W}, 1.7]$ for some references.)

COROLlaRY 5.4. If $\mathbb{Q} \subset A$ then Karoubi's Chern class map factors through a map ch: $K V_{n}(A) \rightarrow H P_{n}(A)$.

COROLlARY 5.5. If $\mathbb{Q} \subset A$ there is a map of homotopy fibrations

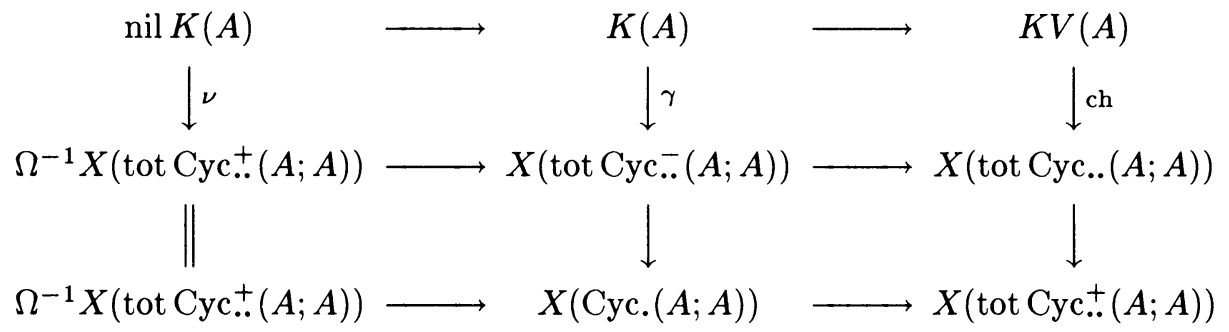

ProOF. The map $\nu$ is induced from $\gamma$ and ch. The bottom two rows are homotopy fibrations by 4.4.1.

DEFINITION 5.6. For $n^{\prime} \geq 1$, let $\nu$ denote $\pi_{n}$ applied to the map $\nu$ of 5.5. Since $\pi_{n} X($ tot Cyc.. $(A ; A))=H C_{n}(A)$, we have defined a map

$$
\nu: \text { nil } K_{n}(A) \rightarrow H C_{n-1}(A) .
$$

REMARK 5.7. If $A$ is not a $\mathbb{Q}$-algebra, we still have the map

$$
\text { nil } K_{n}(A) \rightarrow \operatorname{nil} K_{n}(A \otimes \mathbb{Q}) \stackrel{\nu}{\rightarrow} H C_{n-1}(A \otimes \mathbb{Q}) \cong H C_{n-1}(A) \otimes \mathbb{Q} .
$$

PROOF OF THEOREM C. Diagram 5.5 gives rise to maps of long exact sequences on homotopy. This diagram is the diagram of Theorem C. Q.E.D.

6. Nil $K$-theory. In $\S 5$ we defined groups nil $K_{n}(A)$ fitting into the long exact sequence

$$
\cdots K_{n+1}(A) \rightarrow K V_{n+1}(A) \rightarrow \operatorname{nil} K_{n}(A) \rightarrow K_{n}(A) \rightarrow K V_{n}(A) \cdots .
$$

Note that nil $K_{n}(A)=0$ if $A$ is regular, for then $K_{n}(A)=K V_{n}(A)$. In this section, we establish some of nil $K$ 's properties, including

THEOREM B. If $\mathbb{Q} \subset A$ then nil $K_{n}(A)$ is a $\mathbb{Q}$-vector space for all $n$. If $p^{n}=0$ in $A$ then nil $K_{n}(A)$ is a p-group for all $n$.

EXAMPLE 6.2 [W1 $4.3-4.4$ ]. Let $k \rightarrow K$ be a map of regular rings, and set $B=k \oplus t K[t], C=k \oplus t K[[t]]$. Then $K V_{1}(B) \cong K_{1}(k)$,

$$
\text { nil } K_{1}(B) \cong \text { nil } K_{1}(C) \cong \Omega_{K / k}
$$

and the following sequences are exact:

$$
\begin{gathered}
0 \rightarrow \operatorname{nil} K_{1}(B) \rightarrow K_{1}(B) \rightarrow K V_{1}(B) \rightarrow 0 \\
K_{2}(C) \rightarrow K V_{2}(C) \rightarrow \operatorname{nil} K_{1}(C) \rightarrow 0 .
\end{gathered}
$$


Note that the definition of nil $K_{*}$ in $[\mathbf{W} \mathbf{1}, 4.1]$ is a quotient of the nil $K_{*}$ defined in this paper, so that 6.2 is not in disagreement with [W1,4.4]. Similarly, the remark in [W1] that nil $K_{1}(A)$ is the image of $N K_{1}(A) \rightarrow K_{1}(A)$ becomes incorrect if we use our new definition; there is an exact sequence

$$
N^{2} K_{1}(A) \rightarrow N K_{1}(A) \rightarrow \operatorname{nil} K_{1}(A) \rightarrow 0 .
$$

This is a special case of the following more general result.

THEOREM 6.4. There is a first quadrant spectral sequence, defined for $p \geq$ $1, q \geq 1$,

$$
\begin{aligned}
& E_{p q}^{1}=N^{p} K_{q}(A) \Longrightarrow \operatorname{nil} K_{p+q-1}(A) . \\
& \text {... } \\
& 0 \quad N K_{3} \leftarrow N^{2} K_{3} \\
& 0 \quad N K_{2} \leftarrow N^{2} K_{2} \leftarrow \cdots \\
& 0 \quad N K_{1} \leftarrow N^{2} K_{1} \leftarrow N^{3} K_{1} \cdots \\
& \begin{array}{llll}
0 & 0 & 0 & 0
\end{array}
\end{aligned}
$$

Here $N^{p} K_{q}(A)$ is the intersection of the kernels of the maps

$$
\left(t_{i}=0\right): K_{q}\left(A\left[t_{1}, \ldots, t_{p}\right]\right) \rightarrow K_{q}\left(A\left[t_{1}, \ldots, \hat{t}_{i}, \ldots, t_{p}\right]\right), \quad i=1, \ldots, p,
$$

and the $d^{1}$ map is induced from the ring map

$$
\left(t_{p}=1-\sum t_{i}\right): A\left[t_{1}, \ldots, t_{p}\right] \rightarrow A\left[t_{1}, \ldots, t_{p-1}\right] .
$$

Proof of ThEOREM B. This is immediate from Theorem 6.4 and the fact (q.v. $[\mathbf{W 4}, 3.3]$ ) that the groups $N^{i} K_{q}(A)$ are $\mathbb{Q}$-modules (resp., $p$-groups) when $A$ is an algebra over $\mathbb{Q}$ (resp., some $\mathbb{Z} / p^{n}$ ) and $i \geq 1$.

To establish 6.4 above, we shall construct nil $K(A)$ at the level of simplicial CW spectra, following [W3, §3]. The construction of [W3, 2.2] yields a spectrum $\mathbf{K}(A)$ with $\Omega^{\infty} \mathbf{K}(A) \cong B \mathrm{GL}^{+}(A)$. The simplicial spectrum $\mathbf{K}(\Delta A)$ has total spectrum $|\mathbf{K}(\Delta A)|$ whose homotopy groups yield $K V_{*}(A)$ by $[\mathbf{W 4}, 2.1]$. Now consider the cofibers $\mathbf{K}\left(\Delta_{n} A\right) / \mathbf{K}(A)$; they assemble to form a simplicial spectrum $\mathbf{K}(\Delta A) / \mathbf{K}(A)$. Since cofibrations and fibrations of spectra are the same thing,

$$
\mathbf{K}(A) \rightarrow \mathbf{K}\left(\Delta_{n} A\right) \rightarrow \mathbf{K}\left(\Delta_{n} A\right) / \mathbf{K}(A)
$$

is a homotopy fibration of connected spectra for each $n$. Hence we deduce

LEMMA 6.5. The sequence of total spectra

$$
\mathbf{K}(A) \rightarrow|\mathbf{K}(\Delta A)| \rightarrow|\mathbf{K}(\Delta A) / \mathbf{K}(A)|
$$

is a homotopy fibration for each $n$.

DEFinition 6.6. The spectrum $\Sigma^{-1}|\mathbf{K}(\Delta A) / \mathbf{K}(A)|$ will be denoted nil $\mathbf{K}(A)$ and $\pi_{n}($ nil $\mathbf{K}(A))$ will be denoted nil $K_{n}(A)$. By 6.5 , this agrees with the definition of nil $K_{n}(A)$ given in 5.2 above, and nil $K(A)$ is the space $\Omega^{\infty}$ nil $\mathbf{K}(A)$.

PROOF OF THEOREM 6.4. This follows immediately from [W3, 3.2] applied to the simplicial spectrum $\mathbf{K}(\Delta A) / \mathbf{K}(A)$ with $D=\Sigma^{\infty} S^{0}$.

It is clear from [W3, §3] that variants of Theorem 6.4 and Theorem B hold in relative and doubly relative contexts as well. The following examples illustrate this phenomenon. 
EXAMPLE 6.7. When $I$ is a nilpotent ideal in $A$, we know from [W2, 2.2] that $K V_{n}(A, I)=0$. Hence nil $K_{n}(A, I) \cong K_{n}(A, I)$ for all $n$. Theorem B in this case follows from $[\mathbf{W 3}, 1.4]$ and $[\mathbf{W 4}, 5.4]$.

EXAMPLE 6.8. When $A=A_{0} \oplus A_{1} \oplus \cdots$ is a graded ring, and $I=I_{1} \oplus \cdots$ a graded ideal, we know that $K V_{n}(A, I)=0$ because $K V_{n}(A)=K V_{n}\left(A_{0}\right)$. Hence

$$
\text { nil } K_{n}(A, I) \cong K_{n}(A, I) \text { for all } n \text {. }
$$

Theorem B in this case follows from [W4, 3.7].

EXAMPLE 6.9 (EXCISION). Let $I$ be an ideal of $A$ mapped by $A \rightarrow B$ isomorphically onto an ideal of $B$. Then we can consider the doubly relative $K$-groups. By $[\mathbf{W}, 2.6], K V_{n}(A, B, I)=0$, so

$$
\operatorname{nil} K_{n}(A, B, I) \cong K_{n}(A, B, I) \text { for all } n \text {. }
$$

Theorem $\mathrm{B}$ in this case follows from [W3, 1.5].

EXAMPLE 6.10. Let $A$ be a 1-dimensional noetherian seminormal ring, such as the coordinate ring of a curve with normal crossings. The normalization $B$ of $A$ is Dedekind, and the conductor ideal $I$ is radical in $B$. Hence nil $K_{*}(B)$ $=\operatorname{nil} K_{*}(A / I)=\operatorname{nil} K_{*}(B / I)=0$. It follows that nil $K_{*}(B, I)=0$ and that nil $K_{*}(A, I)=\operatorname{nil} K_{*}(A)$, e.g., from the long exact sequence

$$
\cdots \operatorname{nil} K_{n+1}(A / I) \rightarrow \operatorname{nil} K_{n}(A, I) \rightarrow \operatorname{nil} K_{n}(A) \rightarrow \operatorname{nil} K_{n}(A / I) \cdots .
$$

From the long exact sequence

$$
\cdots \operatorname{nil} K_{n+1}(B, I) \rightarrow \operatorname{nil} K_{n}(A, B, I) \rightarrow \operatorname{nil} K_{n}(A, I) \rightarrow \operatorname{nil} K_{n}(B, I) \cdots
$$

and 6.9 , we see that in this case

$$
\operatorname{nil} K_{n}(A) \cong K_{n}(A, B, I) \text {. }
$$

\section{REFERENCES}

[And] D. W. Anderson, Relationship among K-theories, Lecture Notes in Math., vol. 341, SpringerVerlag, Berlin and New York, 1973.

[Bur] D. Burghelea, The cyclic homology of the group rings, Comment. Math. Helv. 60 (1985), 354-365.

[Bur1] _ , Cyclic homology and the algbraic K-theory of spaces I, Contemp. Math., vol. 55, part I, Amer. Math. Soc., Providence, R.I., 1986.

[C] A. Connes, Cohomologie cyclique et foncteurs Ext ${ }^{n}$, C.R. Acad. Sci. Paris 296 (1983), 953-958.

[CE] H. Cartan and S. Eilenberg, Introduction to homological algebra, Princeton Univ. Press, Princeton, N.J.,1956.

[CK] A. Connes and M. Karoubi, Caractère multiplicatif d'un module de Fredholm, C.R. Acad. Sci. Paris 299 (1984), 963-968.

[D] R. K. Dennis, Algebraic K-theory and Hochschild homology, unpublished notes, 1975-1976.

[DI] R. K. Dennis and K. Igusa, Hochschild homology and the second obstruction for pseudo-isotopy, Lecture Notes in Math., vol. 966, Springer-Verlag, Berlin and New York, 1982.

[G] T. Goodwillie, Algebraic K-theory and cyclic homology, preprint, 1985.

[G1] _ Cyclic homology, derivations, and the free loop space, Topology 24 (1985), 187-215.

[IAS] Institute for Advanced Study, Cyclic homology seminar, 1984-85.

[K] M. Karoubi, Homologie cyclique des groupes et des algèbres, C.R. Acad. Sci. Paris 297 (1983), 381-384.

[K1] _ Homologie cyclique et K-théorie algébrique, C.R. Acad. Sci. Paris 297 (1983), 447-450 and 513-516. 
[K2] _ Homologie cyclique et régulateurs en K-théorie algébrique, C.R. Acad. Sci. Paris 297 (1983), 557-560.

[Kass] C. Kassel, Cyclic homology, comodules and mixed complexes, J. Algebra (to appear).

[L] J.-L. Loday, Symboles en K-théorie algébrique superieure, C.R. Acad. Sci. Paris 292 (1981), 863-866.

[LQ] J.-L. Loday and D. Quillen, Cyclic homology and the Lie algebra homology of matrices, Comment. Math. Helv. 59 (1984), 565-591.

[Mac] S. Mac Lane, Homology, Springer-Verlag, New York, 1963.

[May] J. P. May, Simplicial objects in algebraic topology, Van Nostrand, Princeton, N.J., 1967.

[Ogle] C. Ogle, On the K-theory and cyclic homology of a square-zero ideal. I, J. Pure Appl. Algebra (to appear).

[Ogle1] _ The map from cyclic homology into K-theory, Ph.D. thesis, Brandeis Univ., 1984.

[OW] C. Ogle and C. Weibel, Relative algebraic K-theory and cyclic homology, preprint, 1987.

[S] R. Staffeldt, Rational algebraic K-theory of certain truncated polynomial rings, Proc. Amer. Math. Soc. 95 (1985), 191-198.

[W] C. Weibel, KV-theory of categories, Trans. Amer. Math. Soc. 267 (1981), 621-635.

[W1] _ K- K-theory and analytic isomorphisms, Invent. Math. 61 (1980), 177-197.

[W2] _ Nilpotence and K-theory, J. Algebra 61 (1979), 298-307.

[W3] _ , Mayer-Vietoris sequences and $\bmod p$ K-theory, Lecture Notes in Math., vol. 966, Springer-Verlag, Berlin and New York, 1982.

[W4] _ Mayer-Vietoris sequences and module structures on $N K_{*}$, Lecture Notes in Math., vol. 854, Springer-Verlag, Berlin and New York, 1981.

\section{School of Mathematics, Institute for Advanced Study, Princeton, New JERSEY 08540}

Current address: Department of Mathematics, Rutgers University, New Brunswick, New Jersey 08903 